Article

\title{
In Vivo Assessment of Hypoxia Levels in Pancreatic Tumors Using a Dual-Modality Ultrasound/Photoacoustic Imaging System
}

\author{
Yuhling Wang ${ }^{1,+}{ }^{,}$, De-Fu Jhang ${ }^{1,2,+}$, Chia-Hua Tsai ${ }^{1}$, Nai-Jung Chiang ${ }^{3,4}$, Chia-Hui Tsao ${ }^{1}$, \\ Chiung-Cheng Chuang ${ }^{2}$, Li-Tzong Chen ${ }^{3,5, \ddagger}$, Wun-Shaing Wayne Chang ${ }^{3, *, \ddagger}$ and Lun-De Liao ${ }^{1, *, \neq}$
}

Citation: Wang, Y.; Jhang, D.-F.; Tsai, C.-H.; Chiang, N.-J.; Tsao, C.-H.; Chuang, C.-C.; Chen, L.-T.; Chang, W.-S.W.; Liao, L.-D. In Vivo Assessment of Hypoxia Levels in Pancreatic Tumors Using a Dual-Modality Ultrasound/ Photoacoustic Imaging System. Micromachines 2021, 12, 668. https:// doi.org/10.3390/mi12060668

Academic Editors: Qifa Zhou, Yi Zhang and Huikai Xie

Received: 14 May 2021

Accepted: 3 June 2021

Published: 7 June 2021

Publisher's Note: MDPI stays neutral with regard to jurisdictional claims in published maps and institutional affiliations.

Copyright: (c) 2021 by the authors. Licensee MDPI, Basel, Switzerland. This article is an open access article distributed under the terms and conditions of the Creative Commons Attribution (CC BY) license (https:/ / creativecommons.org/licenses/by/ $4.0 /)$.
1 Institute of Biomedical Engineering and Nanomedicine, National Health Research Institutes, No. 35, Keyan Rd., Zhunan Township, Miaoli 35053, Taiwan; yuhlingwang@nhri.edu.tw (Y.W.); y217834@gmail.com (D.-F.J.); vanessatsai@nhri.edu.tw (C.-H.T.); tsaochiahui@nhri.edu.tw (C.-H.T.)

2 Department of Biomedical Engineering, College of Engineering, Chung Yuan Christian University, No. 200, Chung Pei Road, Chung Li District, Taoyuan 32023, Taiwan; cheng965@cycu.edu.tw

3 National Institute of Cancer Research, National Health Research Institutes, No. 35, Keyan Rd., Zhunan Township, Miaoli 35053, Taiwan; njchiang@nhri.edu.tw (N.-J.C.); leochen@nhri.edu.tw (L.-T.C.)

4 Department of Oncology, National Cheng Kung University Hospital, Tainan 70403, Taiwan

5 Kaohsiung Medical University Hospital, Kaohsiung Medical University, No. 100, Shih-Chuan 1st Road, Sanmin Dist., Kaohsiung 80708, Taiwan

* Correspondence: wayne@nhri.edu.tw (W.-S.W.C.); ldliao@nhri.edu.tw (L.-D.L.)

$+\quad$ Y.W. and D.-F.J. are co-first authors.

$\ddagger \quad$ L.-T.C., W-S.W.C. and L.-D.L. contributed equally.

Abstract: Noninvasive anatomical and functional imaging has become an essential tool to evaluate tissue oxygen saturation dynamics in preclinical or clinical studies of hypoxia. Our dual-wavelength technique for photoacoustic (PA) imaging based on the differential absorbance spectrum of oxyhemoglobin (oxy- $\mathrm{Hb}$ ) and deoxyhemoglobin (deoxy-Hb) can quantify tissue oxygen saturation using the intrinsic contrast property. PA imaging of tissue oxygen saturation can be used to monitor tumorrelated hypoxia, which is a particularly relevant functional parameter of the tumor microenvironment that has a strong influence on tumor aggressiveness. The simultaneous acquisition of anatomical and functional information using dual-modality ultrasound (US) and PA imaging technology enhances the preclinical applicability of the method. Here, the developed dual-modality US/PA system was used to measure relative tissue oxygenation using the dual-wavelength technique. Tissue oxygen saturation was quantified in a pancreatic tumor mouse model. The differences in tissue oxygenation were detected by comparing pancreatic samples from normal and tumor-bearing mice at various time points after implantation. The use of an in vivo pancreatic tumor model revealed changes in hypoxia at various stages of tumor growth. The US/PA imaging data positively correlated with the results of immunohistochemical staining for hypoxia. Thus, our dual-modality US/PA imaging system can be used to reliably assess and monitor hypoxia in pancreatic tumor mouse models. These findings enable the use of a combination of US and PA imaging to acquire anatomical and functional information on tumor growth and to evaluate treatment responses in longitudinal preclinical studies.

Keywords: pancreatic tumor; photoacoustic (PA); ultrasound (US); hypoxia; hemoglobin oxygenation saturation

\section{Introduction}

Hypoxia is a condition present in a wide range of diseases, including stroke [1], pneumonia, cancer [2], sleep apnea [3], etc. Recently, optical imaging techniques, such as diffusion optical imaging (DOI) [4], were used to assess in vivo changes in tissue oxygenation. DOI can be used to directly detect hemoglobin oxygen saturation $\left(\mathrm{SO}_{2}\right)$ signals; however, DOI has insufficient spatial resolution and thus cannot delineate the fine details of 
specific structures due to the diffusive nature of light in biological tissues [4]. Photoacoustic (PA) imaging is a rapidly developing technology based on the optical excitation of the molecules by visible or near-infrared (NIR) range pulsed lasers, resulting in a thermoelastic effect of PA signals [4]. The expansion and contraction caused by the absorption of laser energy generates ultrasound (US) waves that can be detected and reconstructed by the traditional US technique. By utilizing intrinsic optical absorption along with US detection, PA imaging can provide multiscale resolution and deep tissue penetration [4]. Depending on the intrinsic biological optical contrast agent (i.e., blood or melanin), the PA imaging technique offers anatomical and functional information about normal and abnormal tissues and biological phenomena, such as angiogenesis and changes in $\mathrm{SO}_{2}$ and total hemoglobin concentrations [5]. Additionally, some advantages of PA are similar to the advantages of US and optical imaging, such as safety and high spatial resolution [4].

Previously, we developed a functional PA microscopy (fPAM) technique that can preclinically evaluate the changes in functional cerebral blood volume (CBV) and $\mathrm{SO}_{2}$ in the rat cortex in studies of ischemic stroke [6-8]. The dual-wavelength PA technique uses hemoglobin as an intrinsic contrast agent and thus does not require an exogenous contrast agent for blood volume and $\mathrm{SO}_{2}$ imaging [9]. Numerous fPAM applications, especially in preclinical studies, have been gradually developed in recent years [10-13].

In addition to the characterization of ischemic stroke [13], our fPAM system can also be applied to monitoring $\mathrm{SO}_{2}$ in pancreatic cancer. Pancreatic cancer is a lethal disease that has a limited response to cytotoxic chemoradiotherapy $[14,15]$ and even newer immunotherapies [2,3]. The limited success of immunotherapy in pancreatic cancer is likely due to the numerous immunosuppressive pathways upregulated in the hypoxic tumor microenvironment $[16,17]$. Hypoxia in pancreatic tumors is heterogeneous and exacerbates the complex immunosuppressive environment [18]. Hypoxia occurs in most solid tumors because the abnormal vasculature cannot provide adequate oxygen delivery demanded by rapidly proliferating cancer cells [19]. Because tumor hypoxia is a major impediment to effective cancer therapy [20,21], it is important to assess the dynamics of hypoxia in pancreatic tumors in real-time at various tumor stages or in drug treatment studies in preclinical models.

Current common methods for assessing tumor hypoxia in preclinical models include the use of oxygen electrodes [22], imaging of hypoxia biomarkers [23], blood oxygen leveldependent (BOLD) functional magnetic resonance imaging (fMRI) [24,25], and positron emission tomography (PET) [26]. Each technique evaluates various aspects of the hypoxic microenvironment because different aspects provide specific information on hypoxia $[27,28]$. BOLD MRI and fMRI techniques are noninvasive and can be used for multiparametric measurements, including blood oxygenation, blood flow, blood volume, and oxygen metabolism $[29,30]$. BOLD MRI or fMRI techniques do not have tissue depth limitations; however, they cannot be used to investigate transient functional hemodynamic responses in the blood vessels with unambiguous resolution [25,30]. In addition to fMRI, ultrasound imaging is being actively developed [31]. Excellent reviews of these technologies are available in the literature [32,33].

In this study, we aimed to address the feasibility of the investigation of real-time pancreatic tumor oxygenation dynamics using a label-free approach by three-dimensional (3D) PA imaging in parallel with conventional B-mode US imaging. We initially tested our PA imaging system using blood vessel phantoms in vitro. Then, we tested our system by imaging the skin vasculature of a rat in vivo. To assess the ability of our PA imaging system to quantify tissue $\mathrm{SO}_{2}$, we performed a controlled hypoxia study in which hypoxia was induced in vivo through the inhalation of a gas mixture routinely used for anesthesia. After the controlled hypoxia study, we imaged hypoxia in orthotopic pancreatic tumors in vivo. We provided evidence that this combined system can be used to reliably assess the differences in tissue oxygenation between control and tumor-bearing mice using dual-wavelength PA technique to provide structural information at a proper resolution. Im- 
munohistochemical (IHC) staining for hypoxia using pimonidazole confirmed the hypoxia dynamics detected using the developed PA system with the dual-wavelength technique.

\section{Materials and Methods}

\subsection{Dual-Modality US/PA Imaging System}

A diagram of the developed dual-modality US/PA imaging system is shown in Figure 1, including the details of the system block design. The system consisted of a high-frequency 18.5-MHz US transducer (L22-14v, Verasonics, Inc., Kirkland, WA, USA) connected to a 128-channel Verasonics high-frequency ultrasound platform (Vantage 128, Verasonics, Inc., Kirkland, WA, USA) for data acquisition. The US transducer had a -6 $\mathrm{dB}$ fractional bandwidth of $67 \%$. For PA imaging, a compact excitation Nd:YAG laser system was used with an integrated tunable optical parametric oscillator (OPO) system (SpitLight 600 OPO, InnoLas Laser GmbH, Krailling, Germany). The OPO generated approximately 7-ns-long pulses with a repetition rate of $20 \mathrm{~Hz}$ and tunable wavelengths from 680 to $2400 \mathrm{~nm}$. A customized fiber bundle-based illumination system was used to deliver the laser energy to the imaging target from the laser output. This fiber bundle-based illumination system was designed to be $1.5-\mathrm{m}$ long and contained approximately 21,000 leaded glass fibers $50 \mu \mathrm{m}$ in thickness with a high numerical aperture (NA). Each fiber had a core diameter of $47 \mu \mathrm{m}(50 \mu \mathrm{m}$ with cladding) and an NA of 0.66 . The fiber efficiency was estimated to be approximately $60-70 \%$ based on the measurements of the input and output energy at the ends of the fiber bundle-based illumination system [9]. The axial resolution was calculated as the full width at half maximum of each Gaussian function and was previously measured to be $124 \pm 31 \mu \mathrm{m}$ for our PA imaging system [4]. To image in the PA mode, the laser excitation needed to be synchronized with US data acquisition. Synchronization was performed using custom-developed software written in MATLAB (R2007a, MathWorks, Natick, MA, USA). The acoustic waves were received, reconstructed, and displayed on the computer screen at a frame rate of 20 frames per second. The American National Standards Institute (ANSI) safety limit is $20 \mathrm{~mJ} / \mathrm{cm}^{2}$, and the incident energy density on the sample surface during PA imaging was estimated to be approximately 12 $\mathrm{mJ} / \mathrm{cm}^{2}$, which is within the safety limit $[29,34]$.

A customized, precision $3 \mathrm{D}$ translation stage with motorized $\mathrm{x}-, \mathrm{y}-$, and $\mathrm{z}$-axes was used to control the transducer to obtain A-scan, B-scan (i.e., two-dimensional; one axis is the lateral scanning distance, and the other axis is the imaging depth), and C-scan (i.e., three-dimensional) images [9]. Both the phantoms and probe were immersed in a water tank for in vitro imaging. For in vivo imaging, the PA probe was immersed in an acrylic water tank with a rectangular cutout at the bottom serving as an imaging window. The cutout was sealed with a thin polyethylene film 15- $\mu \mathrm{m}$ thick. US gel (POC Medical, Inc., Zhongli City, Taiwan) or an agarose pad was then used to provide a coupling interface between the imaging window and the animal [6]. 


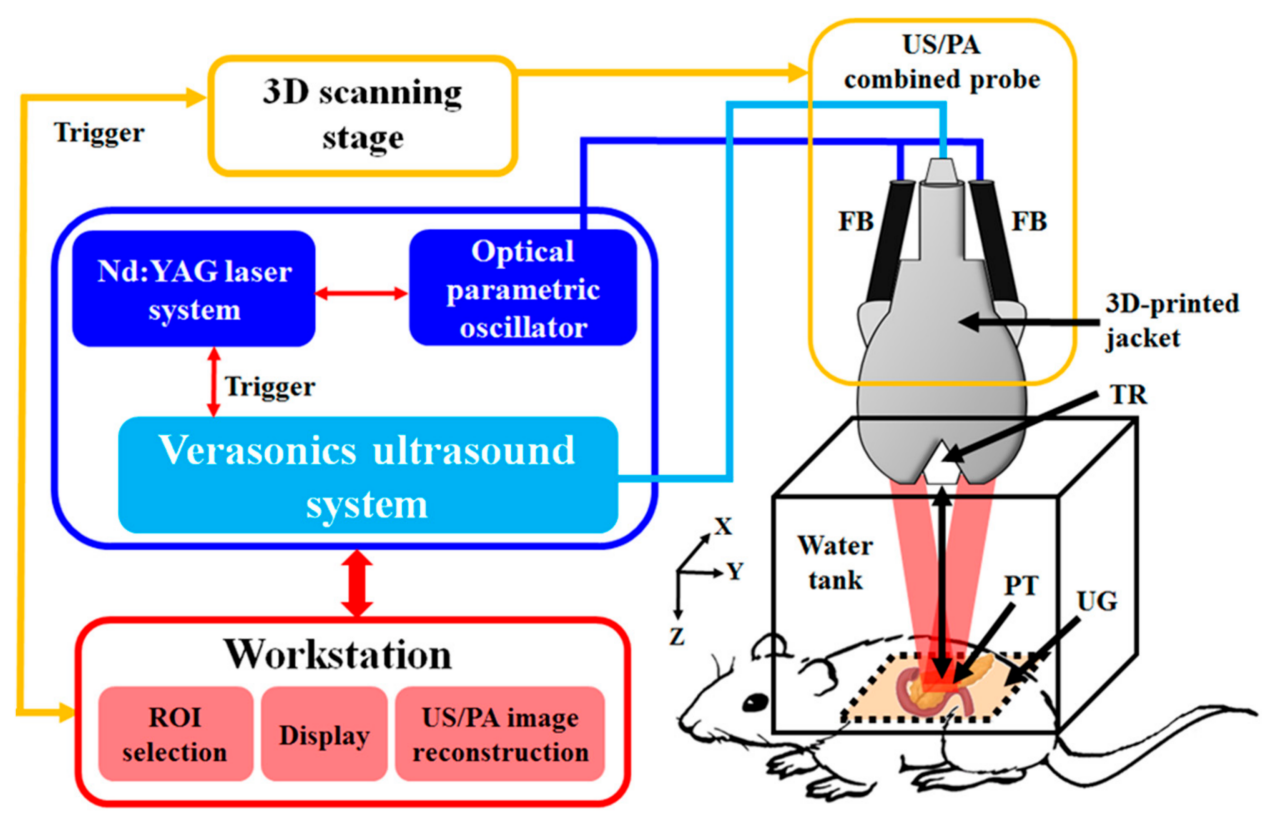

Figure 1. Schematic diagram of the developed dual-modality US/PA imaging system. Using the photoacoustic effect, US waves were generated by delivering light generated by a laser to the imaging target with a pulse repetition rate of $20 \mathrm{~Hz}$ using fiber bundles. The US waves were then detected by a $17.5-\mathrm{MHz}$ transducer and processed through a Verasonics ultrasound system. The developed PA probe includes a removable fiber bundle-based illumination system (FB), a single US transducer (TR), and a customized jacket. Two rectangular parts constituted the output end of the fiber bundle, and the input end was circular in shape. ROI, region of interest; PA, photoacoustic; US, ultrasound; TR, ultrasound transducer; FB, fiber bundle; PT, pancreatic tumor; and UG, ultrasound gel.

\subsection{In Vitro Performance of the US/PA Imaging System}

For in vitro testing of the US/PA imaging system, $6 \%$ Intralipid (optical absorption coefficient of $0.01 \mathrm{~cm}^{-1}$ and optical scattering coefficient of $400 \mathrm{~cm}^{-1}$; Sigma-Aldrich, St. Louis, MO, USA) was mixed in water to mimic the optical diffusion encountered in the tissues during in vivo imaging [30]. Tube phantoms containing blue or red ink to mimic oxygenated and deoxygenated blood were then placed in the Intralipid mixture for imaging. B-scan images of the tubes were obtained at the focal zone of the transducer (10 $\mathrm{mm}$ in depth from the transducer surface) using laser excitation at $750 \mathrm{~nm}$ (strong absorption for red ink) and $850 \mathrm{~nm}$ (strong absorption for blue ink) [9]. C-scan images composed of serial cross-sectional images of the tubes were also obtained at a scanning range of $6 \mathrm{~mm}$ with $0.05 \mathrm{~mm}$ step size.

\subsection{In Vivo PA Imaging of Skin Vasculature}

The skin vasculature was imaged on the back of male Wistar rats (National Laboratory Animal Center, Taiwan) weighing 300-350 g. All animals were housed in a controlled room (temperature, $24 \pm 1{ }^{\circ} \mathrm{C}$; relative humidity, $50-60 \%$ ) with a 12-h light/dark schedule (light cycle beginning at $7 \mathrm{am}$ ) and free access to food and water [12,35]. All experimental procedures, animal care protocols, and protocols requiring ethical oversight were performed in accordance with the guidelines approved by the Institutional Animal Care and Use Committee (IACUC) of the National Health Research Institute (approved protocol number: NHRI-IACUC-107100).

Before imaging the rats, the hair was removed using a hair removal cream (Stellar Beauty Biotech Co., Ltd., Keelung City, Taiwan). The animals were anesthetized by inhalation of $1-3 \%$ isoflurane (Panion \& BF Biotech, Inc., Taipei City, Taiwan) in oxygen [12]. The transducer was set as described previously, including an acrylic water tank, an imaging window, and US gel or an agarose pad as a coupling interface with the rat 
skin. Body temperature was maintained at $37{ }^{\circ} \mathrm{C}$ using a heating pad throughout the experiment. Arterial blood $\mathrm{SO}_{2}$ and heart rate were monitored throughout the experiment using the MouseOX Plus small animal vital signs monitoring system (Starr Life Sciences Corp., Oakmont, PA, USA). B-scan images were obtained at $750 \mathrm{~nm}$ due to the strong absorption by deoxyhemoglobin at that wavelength [9].

\subsection{Use of PA Imaging to Monitor Rat Hindlimb Tissue Oxygenation during the Controlled Hypoxia Study}

Research manuscripts reporting large datasets that are deposited in a publicly available database should specify where the data were deposited and provide the relevant accession numbers. If the accession numbers have not yet been obtained at the time of submission, please state that they will be provided during review. They must be provided prior to publication.

For the controlled hypoxia study, rats $(n=5)$ were anesthetized with $1.5-2 \%$ isoflurane in oxygen, and fur was removed from a hindlimb using hair removal cream. The gas mixture supplying the isoflurane vapor (RWD Life Science, San Diego, CA, USA) was alternated between pure oxygen $\left(100 \% \mathrm{O}_{2}\right)$ to induce hyperoxia and carbogen $\left(5 \% \mathrm{O}_{2}, 5 \%\right.$ $\mathrm{CO}_{2}$, and $90 \% \mathrm{~N}_{2}$ ) to induce hypoxia over 5-min periods [36]. A pulse oximeter (MouseOX Plus, Starr Life Sciences Corp., Oakmont, PA, USA) was clipped onto the paw of the scanned limb to verify body $\mathrm{SO}_{2}$ [36]. For PA imaging, a deoxyhemoglobin-dominant absorption wavelength $(\lambda=750 \mathrm{~nm})$ and an oxyhemoglobin-dominant absorption wavelength $(\lambda=850 \mathrm{~nm})$ were used to acquire the data [6,12]. The following equations were used to calculate $\mathrm{SO}_{2}[6,8,9,12]$ :

$$
\mu_{a}^{\lambda}=\varepsilon_{H b O}^{\lambda}[H b O]+\varepsilon_{H b}^{\lambda}[H b]
$$

where $\varepsilon_{H b}^{\lambda}$ and $\varepsilon_{H b O}^{\lambda}$ are the molar extinction coefficients, [Hb] and [HbO] are the concentrations of deoxy- and oxyhemoglobin, respectively, and $\mu_{a}^{\lambda}$ is the optical absorption coefficient $[6,34]$. The optical absorption coefficient is related to acoustic pressure through the following equation:

$$
\mu_{a}^{\lambda}=\frac{P}{\Gamma F}
$$

where $\Gamma$ is the Grüneisen coefficient [37], $P$ is the acoustic pressure, and $F$ is the optical fluence [5]. Laser energy was monitored using a built-in energy monitor in the OPO system, and optical fluence was assumed to be uniform. [ $\mathrm{Hb}]$ and $[\mathrm{HbO}]$ can then be used to calculate $\mathrm{SO}_{2}$ using the following equation:

$$
\mathrm{SO}_{2}=\frac{[\mathrm{HbO}]}{[\mathrm{HbO}]+[\mathrm{Hb}]}
$$

Statistical significance was assessed using a $t$-test with significance defined as $p<0.05[7,25,32]$.

\subsection{Mouse Pan02 Cells}

The mouse Pan02 cell line was a gift from a collaborator at the National Health Research Institutes of Taiwan (Dr. Wun-Shaing Wayne Chang). The cells were maintained in Dulbecco's modified Eagle medium (Thermo Fisher Scientific, Inc., Waltham, MA, USA) supplemented with $10 \%$ fetal bovine serum and in an incubator at $37{ }^{\circ} \mathrm{C}$ and a constant humidity in $5 \% \mathrm{CO}_{2}$. A $0.05 \%$ trypsin-ethylenediaminetetraacetic acid (EDTA) solution (Thermo Fisher Scientific, Inc., Waltham, MA, USA) was used to detach the cells from the culture dish during passage.

\subsection{Orthotopic Implantation of Pan02 Cells}

Pan02 cells were orthotopically implanted in the pancreas of five-week-old C57B1/6 mice (National Laboratory Animal Center, Taipei City, Taiwan). All experimental procedures and animal care protocols were performed in accordance with the guidelines 
approved by the IACUC of the National Health Research Institute (approved protocol number: NHRI-IACUC-107113-A).

For surgery, the mice were anesthetized by the inhalation of $1-2 \%$ isoflurane in oxygen. Carprofen (5 mg/kg, Zoetis, Inc., Taipei City, Taiwan) was injected subcutaneously for analgesia. Hair around the surgical site was shaved, and the pancreas was exteriorized through a small incision in the left flank. Pan02 cells $\left(0.5 \times 10^{6}\right)$ suspended in $50 \mu \mathrm{L}$ of phosphate-buffered solution (PBS) were injected into the tail of the pancreas using a 28-gauge needle and a syringe [38]. The pancreas was then tucked back into the abdominal cavity, and the incision was closed using wound clips and size 3-0 nylon suture (UNIK Surgical Sutures Mfg. Co., Ltd., New Taipei City, Taiwan).

\subsection{In Vivo Pancreatic Tumor Hypoxia Imaging}

Normal and tumor-bearing mice were imaged on various days after tumor cell implantation. The mice were anesthetized by the inhalation of $1-2 \%$ isoflurane in oxygen and imaged through an acrylic water tank. Laser excitation wavelengths of 750 and $850 \mathrm{~nm}$ were used to acquire C-scan PA images for $\mathrm{SO}_{2}$ evaluation [6]. The scanning range and step size were $10 \mathrm{~mm}$ and $0.05 \mathrm{~mm}$, respectively. To accurately identify the regions of interest (ROIs), US C-scan images were acquired and overlaid with PA images [6].

\subsection{IHC Staining for Tumor Hypoxia}

Pimonidazole (Hypoxyprobe, Inc., Burlington, MA, USA) was injected intraperitoneally at the dose of $60 \mathrm{mg} / \mathrm{kg}$ body weight. The mice were sacrificed $1 \mathrm{~h}$ after the injection, and the tumor tissue was flash-frozen in Surgipath FSC 22 clear frozen section compound (Leica Biosystems Richmond, Inc., Richmond, IL, USA). The tissue was cryosectioned at a thickness of $7 \mu \mathrm{m}$ and fixed in ice-cold acetone (Sigma-Aldrich, St. Louis, MO, USA) for $10 \mathrm{~min}$. The sections were incubated with FITC-Mab1 (clone 4.3.11.3; Hypoxyprobe, Inc., Burlington, MA, USA) overnight at $4{ }^{\circ} \mathrm{C}$ to stain for pimonidazole [39]. The slides were then mounted in Fluoroshield mounting medium with DAPI (SigmaAldrich, St. Louis, MO, USA) and stored at $4{ }^{\circ} \mathrm{C}$. Images of three random fields of view (FOVs) were acquired for each sample using an upright fluorescence microscope (DM2500; Leica Microsystems, Wetzlar, Germany) and a color CCD (DP73; Olympus, MA, USA). ImageJ software was used to quantify the mean fluorescence intensity (MFI) [39], and statistical analysis was performed using one-way analysis of variance (ANOVA) with Tukey's multiple comparison test in GraphPad Prism 6 software (GraphPad Software, San Diego, CA, USA).

\section{Results}

\subsection{In Vitro Performance of the US/PA Imaging System}

To test the ability of the developed PA system to image blood vessels, tube phantoms (Figure 2A) were filled with red and blue ink to mimic oxygenated and deoxygenated blood, respectively. The region marked by a yellow-dashed rectangle was imaged using US/PA. Maximum amplitude projections (MAPs) of a C-scan image composed of serial cross-sectional images of the tubes are shown in Figure 2B. The US, PA and overlaid US/PA MAP images of the vascular phantoms are shown in Figure 2B. The yellow-dashed line marks the location of the cross-sectional scan shown in Figure 2C. At $750 \mathrm{~nm}$, the PA signal of the blue ink was dominant. Because the signal intensity of both ink colors was similar at $850 \mathrm{~nm}$, the proportional difference (PARed = PA850/PA750) between the two excitation wavelengths was calculated to obtain the red ink signal [4]. The resulting overlaid images shown in Figure 2B,C show the optical absorption characteristics of the blood vessel phantoms and provide anatomical and functional information [9]. 


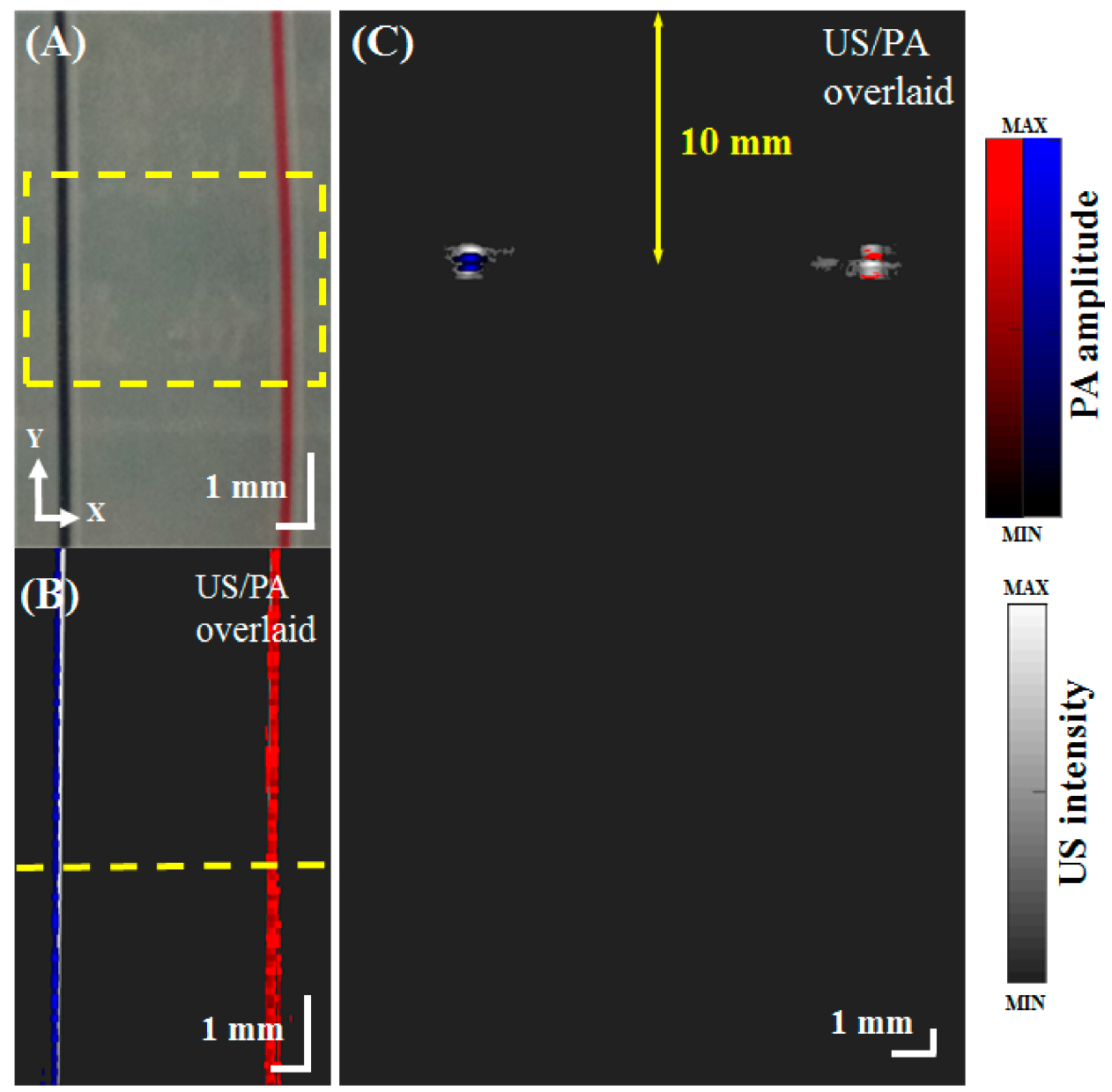

Figure 2. US/PA images of vessel phantoms. (A) Image of the tube phantoms filled with red and blue ink to mimic oxygenated and deoxygenated blood. The phantoms were imaged using US/PA within the yellow dashed rectangle $(3 \mathrm{~mm} \times 6 \mathrm{~mm})$. (B) Overlaid US/PA MAP image (PA850/PA750) of the tube phantoms filled with red and blue ink. The yellow dashed line marks the location of the cross-sectional US/PA B-scan shown in Figure 2C. (C) Overlaid US/PA B-scan image of the phantoms at an imaging depth of $10 \mathrm{~mm}$. PA, photoacoustic; US, ultrasound; PA750, PA signal at an excitation wavelength of $750 \mathrm{~nm}$; PA850, PA signal at an excitation wavelength of $850 \mathrm{~nm}$; and MAP, maximum amplitude projection.

\subsection{In Vivo PA Imaging of Skin Vasculature}

A PA C-scan (3D) image of the skin vasculature on the back of a rat obtained at a wavelength of $750 \mathrm{~nm}$ is shown in Figure 3. MAP matrices containing the peak amplitudes of the PA signals corresponding to absorbing structures on a pixel-by-pixel basis were used to plot the figure [29]. The red lines indicate the position of blood vessels. An area of $12.7 \times 10 \mathrm{~mm}$ with a depth of $1.5 \mathrm{~mm}$ was scanned at a $50-\mu \mathrm{m}$ step size. 


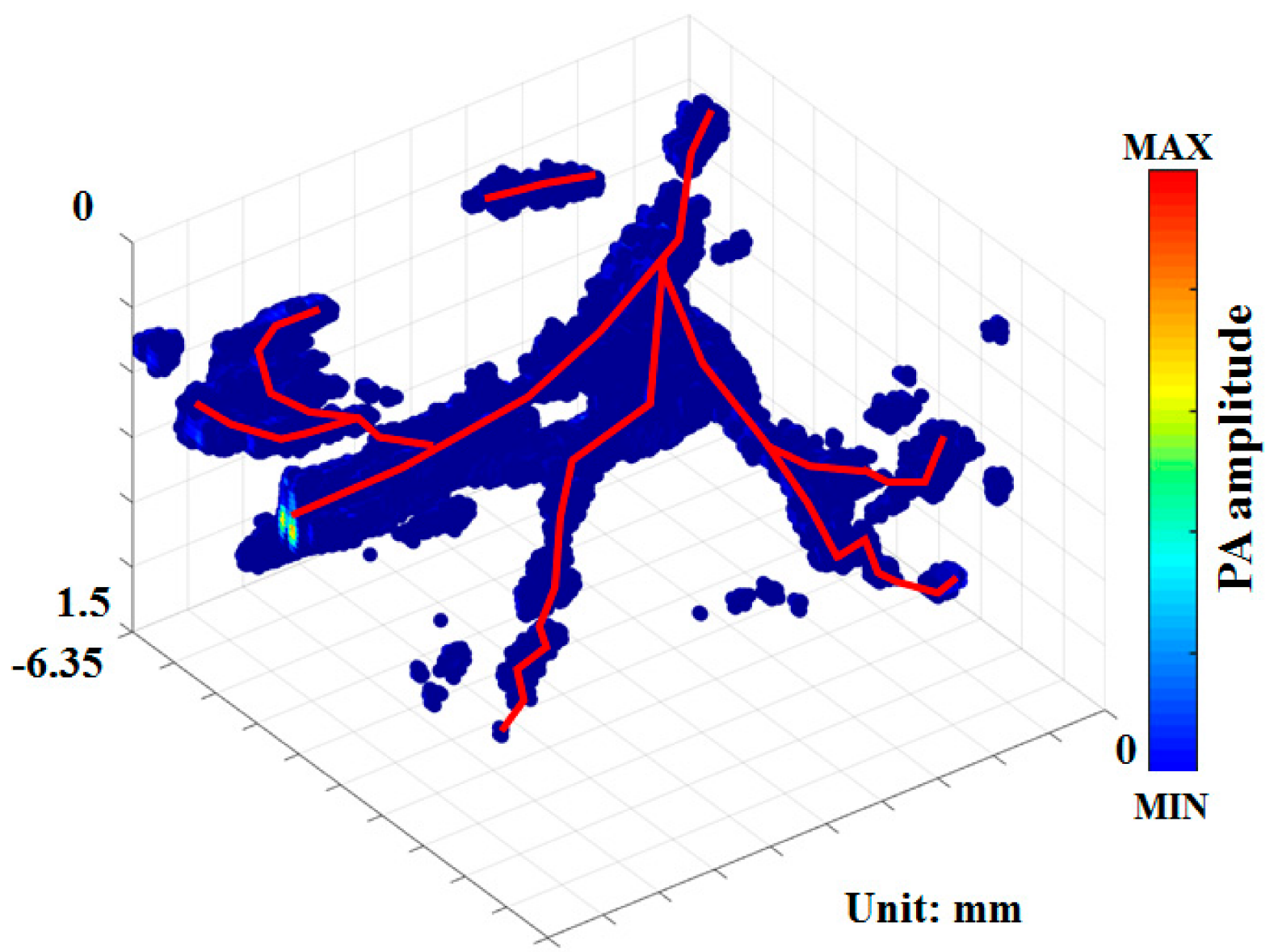

\subsection{0}

Figure 3. PA images of blood vessels acquired by imaging the skin on the back of a rat. Laser excitation at $750 \mathrm{~nm}$ was used to acquire in vivo PA C-scan images. The trends of blood vessels in the skin are indicated by solid red lines.

\subsection{In Vivo PA Monitoring of Rat Hindlimb Tissue Oxygenation during the Hypoxia Challenge}

Many methods have been used to assess hypoxia. The linear model uses multiwavelength PA signals for linear unmixing of oxyhemoglobin and deoxyhemoglobin without compensating for local fluence. Although mathematically simple and easy to implement in most photoacoustic tomography systems, the model is inaccurate at depths exceeding $1 \mathrm{~mm}$ for optical fluence that is independent of wavelength [40-42]. Optical transport models based on Beer's law and diffusion finite-element method can be used to estimate optical fluence distribution in tissue. This model takes into account wavelengthdependent light attenuation. At depths of approximately $10 \mathrm{~mm}$, this model is more accurate than linear models. However, it is not suitable for complex tissue types, such as brain and tumors $[42,43]$. The diffuse optical tomography method mainly evaluates the optical properties of tissue and local optical fluence to correct for the inhomogeneity of the optical fluence when imaging at larger depths $(12 \mathrm{~mm})$. Diffuse optical tomography is a low-resolution imaging modality, and the optical fluence compensation lacks spatial accuracy [44]. In this study, PA signals from different wavelengths were used to quantify the relative hypoxia. The equation used in this study combines molar extinction and optical absorption coefficient and is less sensitive to wavelength associated fluctuations in local optical fluence.

The results for the controlled hypoxia challenge are shown in Figure 4. For Figure 4A-E, the inhaled gas was maintained at $100 \% \mathrm{O}_{2}$ for $10 \mathrm{~min}$. In Figure $4 \mathrm{~A}$, the body $\mathrm{SO}_{2}$ measured using pulse oximetry and $\mathrm{PA} \mathrm{SO}_{2}$ remained at an average of $99.41 \%$ and $56.12 \%$, respectively. As expected, there was no significant change in the tissue oxygen level [36]. 
The PA SO 2 images (Figure 4B-E) collected at 0, 4, 8, and 10 min also showed no changes in $\mathrm{SO}_{2}$. For Figure $4 \mathrm{~F}-\mathrm{J}$, the inhaled gas was maintained at $100 \% \mathrm{O}_{2}$ for $5 \mathrm{~min}$ before switching to the carbogen mixture for another $5 \mathrm{~min}$ to induce hypoxia. Figure $4 \mathrm{~F}$ shows a significant decrease in both body and $\mathrm{PA} \mathrm{SO}_{2}$. The body $\mathrm{SO}_{2}$ was significantly decreased from an average of $99.7 \%$ to $38.74 \%$, whereas the $\mathrm{PA} \mathrm{SO}_{2}$ was decreased from an average of $58.8 \%$ to $35.5 \%$. The $\mathrm{PA} \mathrm{SO}_{2}$ images shown in Figure 4G-J indicate that the oxygenated area (red) decreased after the switch to carbogen, while the ischemic area (blue) increased. These results demonstrate that the assessment of tissue $\mathrm{SO}_{2}$ using our PA imaging system reflects the changes in body $\mathrm{SO}_{2}$ measured using pulse oximetry during hypoxia challenge. A study by Xing et al. showed that changes in hemoglobin oxygen saturation and carboxyhemoglobin saturation could be observed in blood vessels using PA microscopy during carbon monoxide challenge [45]. The resolution of our US/PA imaging system does not allow for observation of single blood vessels but allows for monitoring of larger areas of tissue. After evaluation of hypoxia in a controlled in vivo setting, our next step was to measure hypoxia in the pancreatic tissue of normal and tumor-bearing mice 9.
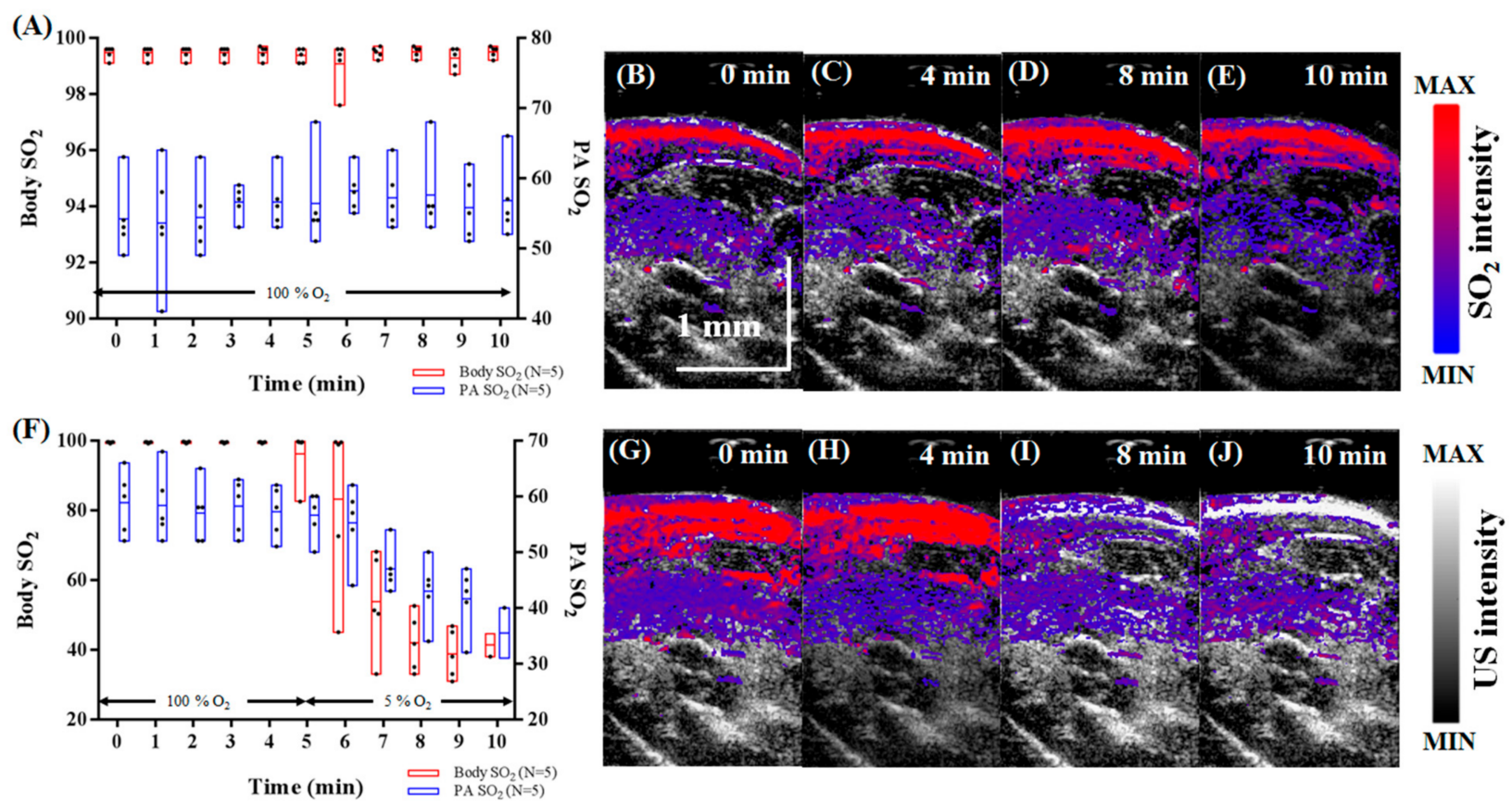

Figure 4. Dynamic changes in $\mathrm{SO}_{2}$ during a controlled hypoxia challenge. (A) Changes in body and $\mathrm{PA} \mathrm{SO}_{2}$ as inhaled gas was maintained at $100 \% \mathrm{O}_{2}$. (B-E) Representative PA images collected over 10 min showing tissue oxygen distribution in the hindlimb were overlaid with US images. (F) Changes in body and $\mathrm{PA} \mathrm{SO}_{2}$ as inhaled gas was switched from $100 \% \mathrm{O}_{2}$ to carbogen at the 5 min mark to induce hypoxia. (G-J) Representative PA/US images collected during hypoxia challenge $\left(\mathrm{N}=5 ;{ }^{*} p<0.05\right)$.

\subsection{In Vivo Imaging of the Changes in Pancreatic Tissue Oxygenation in Normal vs. Tumor-Bearing Mice}

To compare tissue oxygenation in normal vs. tumor-bearing mice, we imaged $\mathrm{SO}_{2}$ levels in the pancreas of normal mice (Figure 5), mice 14 days after tumor implantation (Figure 6), and mice 21 days after tumor implantation (Figure 7). PA B-scan images acquired at $750 \mathrm{~nm}$ (Figures 5A,E,I, 6A,E,I,M and 7A,E,I,M) and $850 \mathrm{~nm}$ (Figures 5B,F, , 6B,F,J,N and $7 \mathrm{~B}, \mathrm{~F}, \mathrm{~J}, \mathrm{~N}$ ) were used to calculate $\mathrm{SO}_{2}$ (Figures $5 \mathrm{C}, \mathrm{G}, \mathrm{K}, 6 \mathrm{C}, \mathrm{G}, \mathrm{K}, \mathrm{O}$ and $7 \mathrm{C}, \mathrm{G}, \mathrm{K}, \mathrm{O}$ ). The 3D C-scan images (Figures 5D,H,L, 6D,H,L,P and 7D,H,L,P) were generated by combining B-scan images. The corresponding rotational 3D images can be seen in Supplementary Materials Videos S1-S3. 


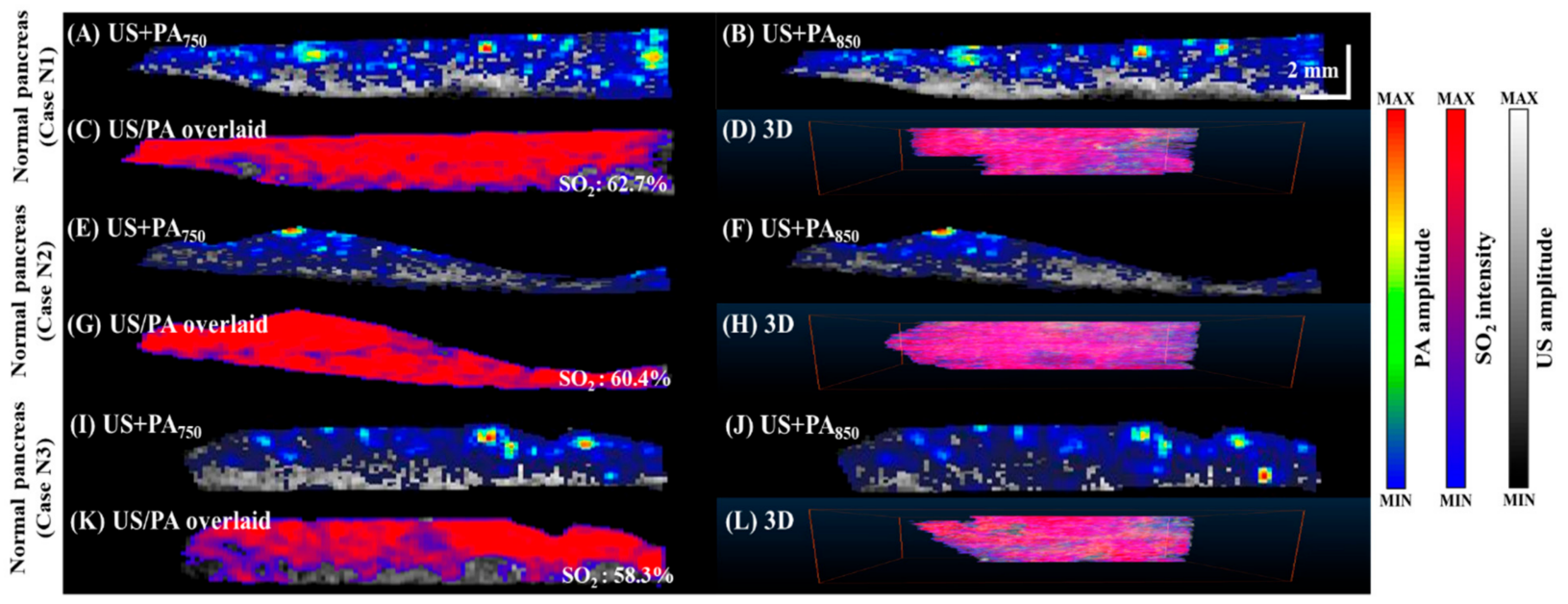

Figure 5. Relative $\mathrm{SO}_{2}$ levels in normal pancreatic regions in three different mice. $\mathrm{SO}_{2}$ was calculated as the percentage of oxygenated hemoglobin relative to total hemoglobin based on dual-wavelength PA imaging at 750 and $850 \mathrm{~nm}$. (A,E,I) PA images acquired at $750 \mathrm{~nm}$ overlaid with grayscale US B-mode images. (B,F,J) PA images acquired at $850 \mathrm{~nm}$ overlaid with grayscale US B-mode images. $(\mathbf{C}, \mathbf{G}, \mathbf{K})$ Calculated $\mathrm{SO}_{2}$ dynamics overlaid with grayscale US B-mode images. $(\mathbf{D}, \mathbf{H}, \mathbf{L}) \mathrm{C}$-scan image of $\mathrm{SO}_{2}$ dynamics overlaid with US. Rotational 3D images can be seen in Supplementary Materials Video S1.

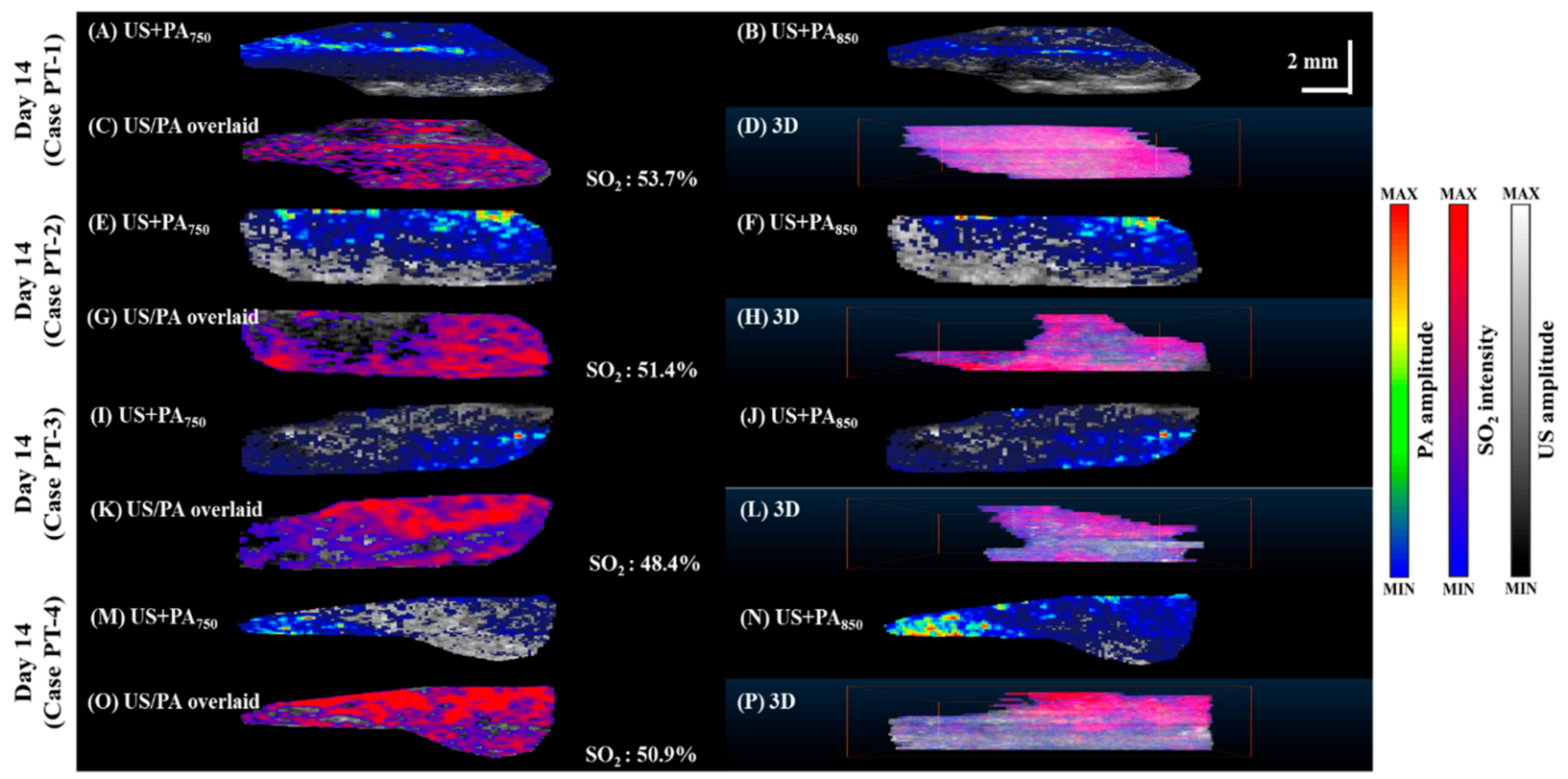

Figure 6. Relative $\mathrm{SO}_{2}$ levels in pancreatic tumor in mice imaged 14 days after the tumor cell injection. (A,E,I,M) PA images acquired at $750 \mathrm{~nm}$ overlaid with grayscale US B-mode images. (B,F,J,N) PA images acquired at $850 \mathrm{~nm}$ overlaid with grayscale US B-mode images. $(\mathbf{C}, \mathbf{G}, \mathbf{K}, \mathbf{O}) \mathrm{SO}_{2}$ was calculated as the percentage of oxygenated hemoglobin relative to total hemoglobin based on dual-wavelength PA imaging at 750 and $850 \mathrm{~nm}$ and overlaid with grayscale US B-scan images. $(\mathbf{D}, \mathbf{H}, \mathbf{L}, \mathbf{P})$ C-scan of $\mathrm{SO}_{2}$ dynamics. Rotational 3D images can be seen in Supplementary Materials Video S2. 


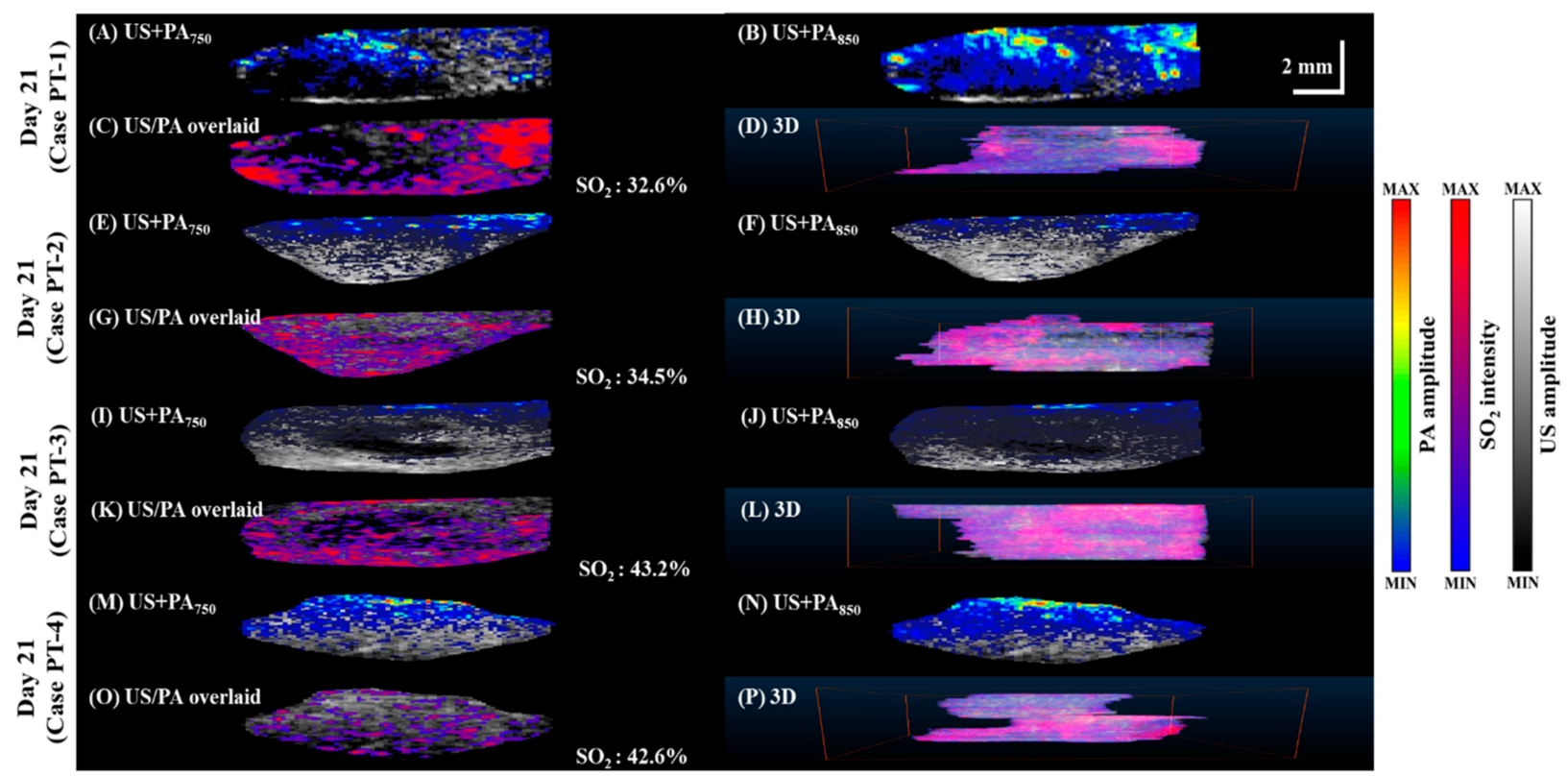

Figure 7. Relative $\mathrm{SO}_{2}$ levels in pancreatic tumor in mice imaged 21 days after tumor cell injection. (A,E,I,M) PA images acquired at $750 \mathrm{~nm}$ overlaid with grayscale US B-mode images. (B,F,J,N) PA images acquired at $850 \mathrm{~nm}$ overlaid with grayscale US B-mode images. $(\mathbf{C}, \mathbf{G}, \mathbf{K}, \mathbf{O}) \mathrm{SO}_{2}$ was calculated as the percentage of oxygenated hemoglobin relative to total hemoglobin based on dual-wavelength PA imaging at 750 and $850 \mathrm{~nm}$ and overlaid with grayscale US B-scan images. $(\mathbf{D}, \mathbf{H}, \mathbf{L}, \mathbf{P})$ C-scan of $\mathrm{SO}_{2}$ dynamics. Rotational 3D images can be seen in Supplementary Materials Video S3.

Figure 8 shows a comparison of the relative $\mathrm{SO}_{2}$ levels in the pancreas of normal and tumor-bearing mice on day 14 and day 21. Representative images selected from C-scan imaging of $\mathrm{SO}_{2}$ levels in normal, day 14, and day 21 mice are shown in Figure 8A-C,D-F, and G-I, respectively. Quantification of the levels of deoxy vs. oxyhemoglobin are shown in Figure 8J-L. In normal mice, the percentage of oxyhemoglobin was significantly greater than the percentage of deoxyhemoglobin (Figure 8J). In contrast, the deoxyhemoglobin levels were significantly higher in day 21 tumor-bearing mice (Figure 8L), suggesting a hypoxic environment. Comparison of tissue $\mathrm{SO}_{2}$ levels between the three groups of mice (Figure 8M) shows significant differences, and a downward trend is seen from normal to day 14 to day 21.

Currently, most preclinical PA imaging systems are used in preclinical studies to assess the tumor microenvironment, hypoxia, angiogenesis, and metastasis [46]. Our device for measuring real-time PA signals uses hemoglobin as an endogenous contrast agent with absorption wavelengths of 750 and $850 \mathrm{~nm}$ to detect $\mathrm{SO}_{2}$ and vascular blood volume at a wavelength of $800 \mathrm{~nm}$ [36] in pancreatic tumors. Previously, hemoglobin was used in PA imaging of brain, breast, and prostate malignancies [4,32,33]; however, data on the dynamic changes in pancreatic cancer models in vivo are limited. Hypoxia within solid tumors is often responsible for the failure of chemotherapy or radiotherapy. The generation of a hypoxic environment and activation of its main effector, hypoxia-inducible factor-1 (HIF-1), leads to tumor progression in advanced cancer [44]. 


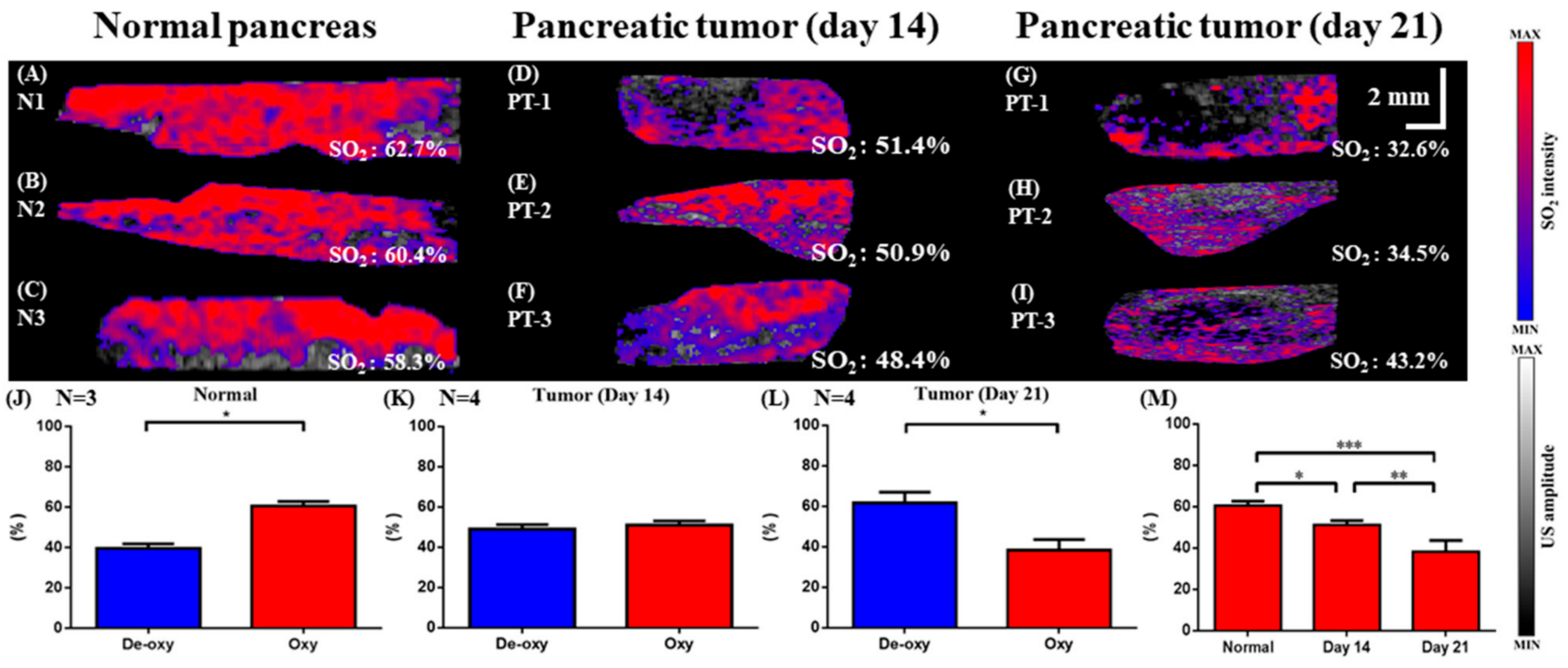

Figure 8. Comparison of the relative $\mathrm{SO}_{2}$ levels in the pancreas of normal and tumor-bearing mice on day 14 and day 21. (A-C) Representative images of $\mathrm{SO}_{2}$ levels in normal mice. (D-F) Representative images of $\mathrm{SO}_{2}$ levels in tumor-bearing mice on day 14 after the tumor cell injection and on day 21 (G-I). (J) Quantification of the levels of deoxy vs. oxyhemoglobin in normal and tumor-bearing mice on day $14(\mathbf{K})$ and day $21(\mathbf{L})$. (M) Comparison of tissue $\mathrm{SO}_{2}$ in normal, day 14, and day 21 tumors $\left({ }^{*} p<0.05,{ }^{* *} p<0.01,{ }^{* * *} p<0.001\right)$.

\subsection{PA Imaging of Pancreatic Tumor Hypoxia Dynamics Is Confirmed by IHC Staining for Pimonidazole}

To confirm the results of the PA measurements of $\mathrm{SO}_{2}$ in pancreatic tumors, IHC staining for hypoxia using pimonidazole was used to compare the samples of the normal pancreas $(n=6)$, pancreatic tumors $<21$ days after the injection $(n=5)$ and pancreatic tumors $\geq 21$ days after the injection $(n=5)$; representative images of the samples after pimonidazole staining are shown in Figure 9A-C. Quantification of MFI in Figure 9D shows a significant increase in pimonidazole staining in the pancreatic tumor samples compared with that in the normal pancreatic samples. Tumors $\geq 21$ days after the injection also exhibited significantly greater staining. This increase in hypoxia is in agreement with the results of the dual-wavelength PA measurement, which showed a decrease in tissue $\mathrm{SO}_{2}$ in tumors $\geq 21$ days after injection compared with tumors $<21$ days after the injection (unpaired $t$-test, $p \leq 0.001$ ). There was high agreement between the histological evaluation of hypoxia in the tumor microenvironment and qualitative and quantitative results of the PA signal analysis, indicating that our device is valid for real-time hypoxia assessment.

Nanodrugs, such as nab-paclitaxel and nanoliposomal irinotecan, have been widely used in the treatment of pancreatic adenocarcinoma [47]. Nanoparticles responsible for enhancing oxygen levels within the tumor and sensitizing hypoxic areas due to the suppression of hypoxia-inducing factors can lead to a decrease in tumor hypoxia and thus enhance the therapeutic efficacy of chemotherapy and immunotherapy [48-51]. In future studies, we will test therapeutic drugs for pancreatic cancer by comparing a parental drug and nanoparticle-based formulations and monitoring the dynamic changes in hypoxia in tumor tissue using our non-invasive real-time dual-modality PA/US system. These efforts will lead to improvements in the design of future clinical trials, expand the perspective to include the molecular and histological implications of novel treatment paradigms, and ultimately change the lives of patients. 

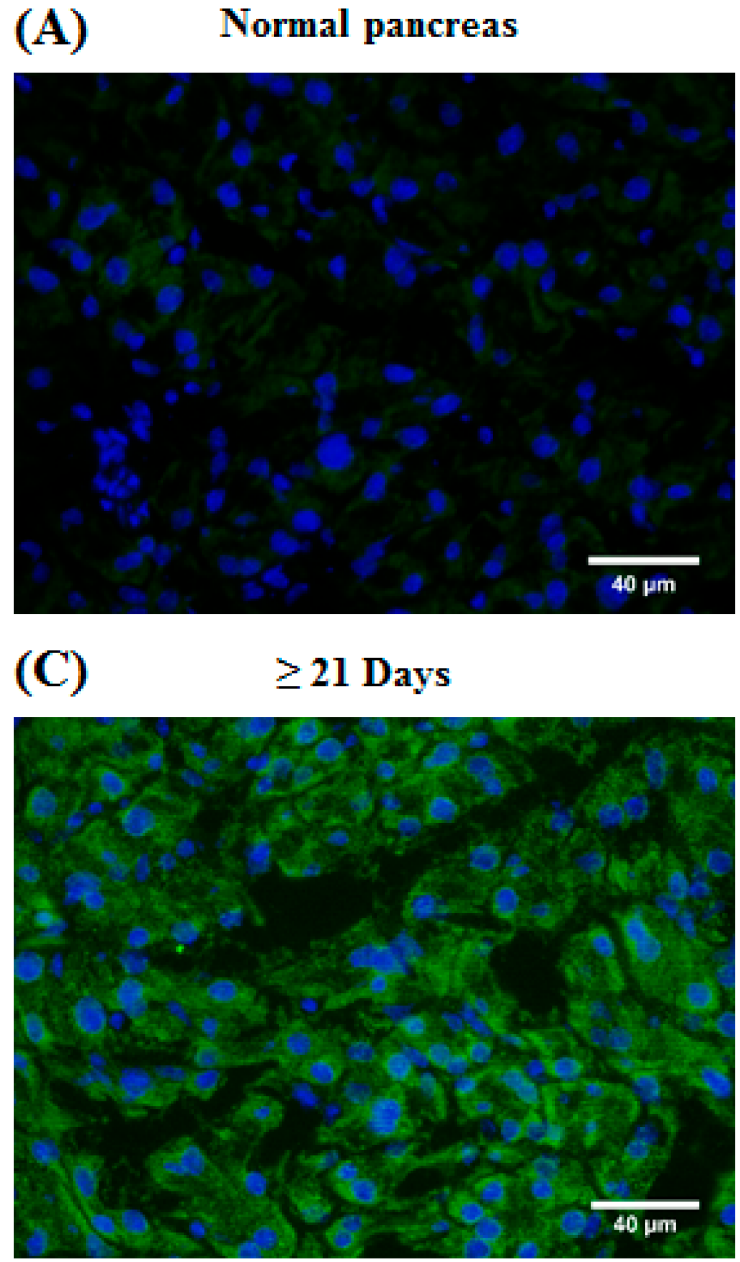

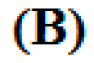
$<21$ Days

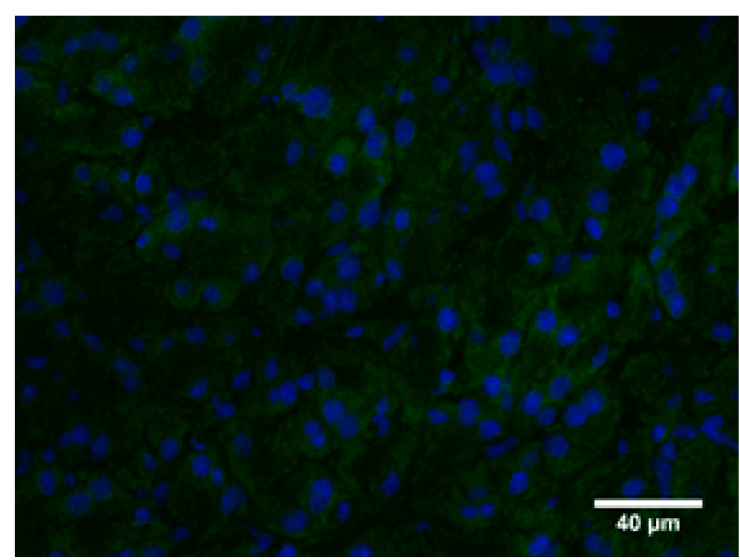

(D)

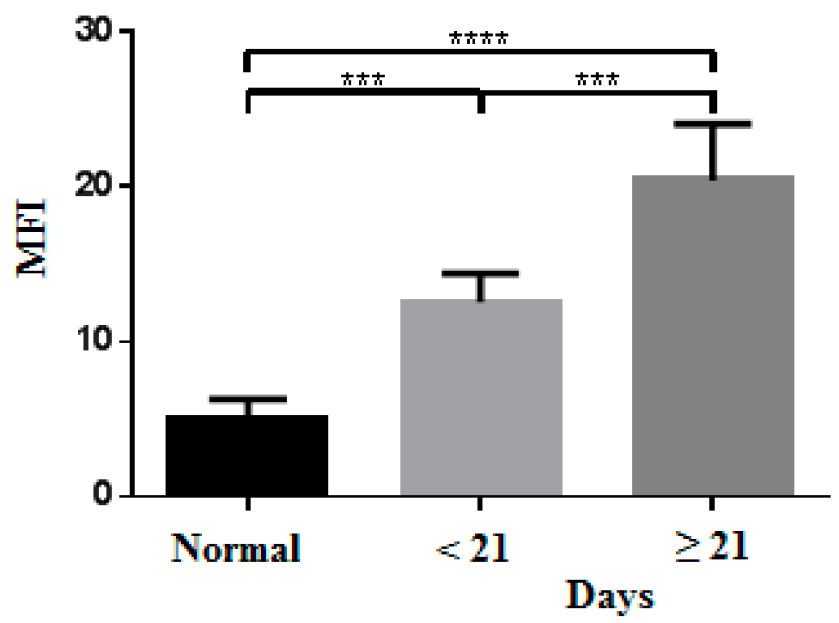

Figure 9. (A-C) Representative images of IHC staining for hypoxia using pimonidazole in the samples of normal pancreas and pancreatic tumors on various days after the tumor cell injection. The nucleus is presented in blue, and areas that stained positive for pimonidazole are shown in green. (D) Quantification of the MFI of pimonidazole staining. ( $\mathrm{n}=6 \mathrm{for}$ normal pancreas, $\mathrm{n}=5$ for $<21$ days, $\mathrm{n}=4$ for $\geq 21$ days; ${ }^{* * *} p \leq 0.001$, ${ }^{* * * *} p \leq 0.0001$ ).

\section{Discussion}

In this study, we present a dual-modality US/PA imaging system that can be used to assess tissue oxygenation in vivo. We initially tested our system with ink-filled phantoms to mimic the in vivo vasculature. Then, we demonstrated that the system could image the vasculature in rat skin. To assess whether our system can quantify tissue oxygenation, the next step involved a controlled hypoxia challenge. The results showed that our system is comparable to pulse oximetry, while having the advantage of presenting spatial information. Then, we imaged the pancreas of normal and tumor-bearing mice. The results demonstrated that our dual-wavelength PA technique, based on the measurement of oxygenated and deoxygenated hemoglobin, yields reliable real-time hypoxia data in vivo in an orthotopic pancreatic tumor mouse model. The $\mathrm{PA} \mathrm{SO}_{2}$ measurements were confirmed by IHC staining for hypoxia, thus validating the applicability of PA for monitoring the functional aspects of tumorigenesis. A previous study by Nania et al. also demonstrated correlation between PA measurement of $\mathrm{SO}_{2}$ and IHC staining for hypoxia in mouse tumor models [52]. The developed dual-wavelength PA technique can be widely used because it does not require an exogenous contrast agent. The results of this study indicate the feasibility of real-time dual-wavelength PA imaging of a preclinical pancreatic tumor mouse model to assess tissue hypoxia. In future studies, the developed dual-wavelength PA imaging 
system can potentially be used to investigate the relationships between hemodynamics and specific biomarkers associated with tumor progression and to evaluate the therapeutic efficacy of treatment.

Supplementary Materials: The following are available online at https:/ / www.mdpi.com/article/10 .3390/mi12060668/s1, Video S1 for Figure 5, Video S2 for Figure 6 and Video S3 for Figure 7.

Author Contributions: Conceptualization, Y.W., L.-T.C., W.-S.W.C. and L.-D.L.; Funding acquisition, L.-D.L.; Investigation, Y.W., D.-F.J., C.-H.T. (Chia-Hua Tsai), C.-H.T. (Chia-Hui Tsao) and L.-D.L.; Methodology, D.-F.J.; Project administration, L.-T.C., W.-S.W.C. and L.-D.L.; Resources, C.-C.C.; Supervision, L.-T.C. and W.-S.W.C.; Validation, Y.W.; Visualization, Y.W.; Writing-original draft, Y.W., D.-F.J., N.-J.C., L.-T.C., W.-S.W.C. and L.-D.L.; Writing-review \& editing, L.-D.L. All authors have read and agreed to the published version of the manuscript.

Funding: This research was funded by the Ministry of Science and Technology of Taiwan (grant numbers 107-2221-E-400-002-MY3, 107-3111-Y-043-012, 108-2314-B-400-025, 108-2221-E-400-003-MY3 and 109-2314-B-400-037); by the National Health Research Institutes of Taiwan (grant numbers CA-108-PP-15, NHRI-EX108-10829EI, NHRI-EX109-10829EI and NHRI-EX110-10829EI); and by the Central Government S \& T grant, Taiwan (grant numbers MR-110-GP-13, 106-0324-01-10-05, 107-032401-19-02 and 108-0324-01-19-06); and by the Ministry of Economic Affairs, Taiwan (grant number 110-EC-17-A-22-1650).

Institutional Review Board Statement: The study was conducted according to the guidelines of the Declaration of Helsinki, and approved by the Institutional Animal Care and Use Committee of the National Health Research Institutes of Taiwan (NHRI-IACUC-107100, approval date 08/01/2018; NHRI-IACUC-107113, approval date 06/30/2018).

Informed Consent Statement: Not applicable.

Data Availability Statement: Data will be provided on request through the corresponding author (Lun-De Liao) of this article.

Conflicts of Interest: The authors declare no conflict of interest.

\section{References}

1. Ferdinand, P.; Roffe, C. Hypoxia after stroke: A review of experimental and clinical evidence. Exp. Transl. Stroke Med. 2016, 8, 1-8. [CrossRef] [PubMed]

2. Jahanban-Esfahlan, R.; de la Guardia, M.; Ahmadi, D.; Yousefi, B. Modulating tumor hypoxia by nanomedicine for effective cancer therapy. J. Cell Physiol. 2018, 233, 2019-2031. [CrossRef] [PubMed]

3. Labarca, G.; Gower, J.; Lamperti, L.; Dreyse, J.; Jorquera, J. Chronic intermittent hypoxia in obstructive sleep apnea: A narrative review from pathophysiological pathways to a precision clinical approach. Sleep Breath. 2020, 24, 751-760. [CrossRef] [PubMed]

4. Liao, L.-D.; Tsytsarev, V.; Delgado-Martinez, I.; Li, M.-L.; Erzurumlu, R.; Vipin, A.; Orellana, J.; Lin, Y.-R.; Lai, H.-Y.; Chen, Y.-Y.; et al. Neurovascular coupling: In vivo optical techniques for functional brain imaging. Biomed. Eng. Online 2013, 12, 38. [CrossRef]

5. Zhu, Y.; Feng, T.; Cheng, Q.; Wang, X.; Du, S.; Sato, N.; Yuan, J.; Singh, M.K.A. Towards Clinical Translation of LED-Based Photoacoustic Imaging: A Review. Sensors 2020, 20, 2484.

6. Liao, L.-D.; Li, M.-L.; Lai, H.-Y.; Shih, Y.-Y.; Lo, Y.-C.; Tsang, S.; Chao, P.C.-P.; Lin, C.-T.; Jaw, F.-S.; Chen, Y.-Y. Imaging brain hemodynamic changes during rat forepaw electrical stimulation using functional photoacoustic microscopy. Neuroimage 2010, 52, 562-570. [CrossRef]

7. Liu, Y.H.; Liao, L.D.; Tan, S.S.H.; Kwon, K.Y.; Ling, J.M.; Bandla, A.; Shih, Y.-Y.I.; Tan, E.T.W.; Li, W.; Ng, W.H. Assessment of neurovascular dynamics during transient ischemic attack by the novel integration of micro-electrocorticography electrode array with functional photoacoustic microscopy. Neurobiol. Dis. 2015, 82, 455-465. [CrossRef]

8. Liao, L.D.; Lin, C.T.; Shih, Y.Y.; Lai, H.Y.; Zhao, W.T.; Duong, T.Q.; Zhao, W.-T.; Li, M.-L. Investigation of the cerebral hemodynamic response function in single blood vessels by functional photoacoustic microscopy. J. Biomed. Opt. 2012, 17, 061210. [CrossRef]

9. Leng, H.; Wang, Y.; Jhang, D.F.; Chu, T.S.; Tsao, C.H.; Tsai, C.H.; Giamundo, S.; Chen, Y.-Y.; Liao, K.-W.; Chuang, C.-C.; et al. Characterization of a Fiber Bundle-Based Real-Time Ultrasound/Photoacoustic Imaging System and Its In Vivo Functional Imaging Applications. Micromachines 2019, 10, 820. [CrossRef]

10. Wang, L.V.; Hu, S. Photoacoustic tomography: In vivo imaging from organelles to organs. Science 2012, 335, 1458-1462. [CrossRef]

11. Beard, P. Biomedical photoacoustic imaging. Interface Focus 2011, 1, 602-631. [CrossRef]

12. Liao, L.D.; Liu, Y.H.; Lai, H.Y.; Bandla, A.; Shih, Y.Y.; Chen, Y.Y.; Thakor, N.V. Rescue of cortical neurovascular functions during the hyperacute phase of ischemia by peripheral sensory stimulation. Neurobiol. Dis. 2015, 75, 53-63. [CrossRef] 
13. Liao, L.D.; Bandla, A.; Ling, J.M.; Liu, Y.H.; Kuo, L.W.; Chen, Y.Y.; King, N.K.K.; Lai, H.-I.; Lin, Y.-R.; Thakor, N.V. Improving neurovascular outcomes with bilateral forepaw stimulation in a rat photothrombotic ischemic stroke model. Neurophotonics 2014, 1, 011007. [CrossRef]

14. Pereira, S.P.; Oldfield, L.; Ney, A.; Hart, P.A.; Keane, M.G.; Pandol, S.J.; Li, D.; Greenhalf, W.; Jeon, C.Y.; Koay, E.J.; et al. Early detection of pancreatic cancer. Lancet. Gastroenterol. Hepatol. 2020, 5, 698-710. [CrossRef]

15. Weibel, P.; Pavic, M.; Lombriser, N.; Gutknecht, S.; Weber, M. Chemoradiotherapy after curative surgery for locally advanced pancreatic cancer: A 20-year single center experience. Surg. Oncol. 2021, 36, 36-41. [CrossRef]

16. Liu, Q.; Liao, Q.; Zhao, Y. Chemotherapy and tumor microenvironment of pancreatic cancer. Cancer Cell Int. 2017, 17, 68. [CrossRef]

17. Gan, L.L.; Hii, L.W.; Wong, S.F.; Leong, C.O.; Mai, C.W. Molecular Mechanisms and Potential Therapeutic Reversal of Pancreatic Cancer-Induced Immune Evasion. Cancers 2020, 12, 1872. [CrossRef]

18. Daniel, S.K.; Sullivan, K.M.; Labadie, K.P.; Pillarisetty, V.G. Hypoxia as a barrier to immunotherapy in pancreatic adenocarcinoma. Clin. Transl. Med. 2019, 8, 10. [CrossRef]

19. Semenza, G.L. HIF-1 and tumor progression: Pathophysiology and therapeutics. Trends Mol. Med. 2002, 8, S62-S67. [CrossRef]

20. Dhani, N.; Fyles, A.; Hedley, D.; Milosevic, M. The Clinical Significance of Hypoxia in Human Cancers. Semin. Nucl. Med. 2015, 45, 110-121. [CrossRef]

21. Otrock, Z.K.; Hatoum, H.A.; Awada, A.H.; Ishak, R.S.; Shamseddine, A.I. Hypoxia-inducible factor in cancer angiogenesis: Structure, regulation and clinical perspectives. Crit Rev. Oncol. Hematol. 2009, 70, 93-102. [CrossRef]

22. Nordsmark, M.; Loncaster, J.; Aquino-Parsons, C.; Chou, S.C.; Ladekarl, M.; Havsteen, H.; Lindegaard, J.C.; Davidson, S.E.; Varia, M.; West, C.; et al. Measurements of hypoxia using pimonidazole and polarographic oxygen-sensitive electrodes in human cervix carcinomas. Radiother. Oncol. 2003, 67, 35-44. [CrossRef]

23. Le, Q.T.; Courter, D. Clinical biomarkers for hypoxia targeting. Cancer Metastasis Rev. 2008, 27, 351-362. [CrossRef]

24. Loued-Khenissi, L.; Doll, O.; Preuschoff, K. An Overview of Functional Magnetic Resonance Imaging Techniques for Organizational Research. Organ. Res. Methods 2019, 22, 17-45. [CrossRef]

25. Glover, G.H. Overview of Functional Magnetic Resonance Imaging. Neurosurg. Clin. North. Am. 2011, 22, 133-139. [CrossRef]

26. Bentzen, L.; Keiding, S.; Nordsmark, M.; Falborg, L.; Hansen, S.B.; Keller, J.; Nielsen, O.S.; Overgaard, J. Tumour oxygenation assessed by $18 \mathrm{~F}-$ fluoromisonidazole PET and polarographic needle electrodes in human soft tissue tumours. Radiother. Oncol. 2003, 7, 339-344. [CrossRef]

27. Rankin, E.B.; Giaccia, A.J. Hypoxic control of metastasis. Science 2016, 352, 175-180. [CrossRef]

28. Nizet, V.; Johnson, R.S. Interdependence of hypoxic and innate immune responses. Nat. Rev. Immunol. 2009, 9, 609-617. [CrossRef]

29. Kim, S.G.; Ogawa, S. Biophysical and physiological origins of blood oxygenation level-dependent fMRI signals. J. Cereb. Blood Flow Metab. 2012, 32, 1188-1206. [CrossRef]

30. Matthews, P.M.; Jezzard, P. Functional magnetic resonance imaging. J. Neurol. Neurosurg. Psychiatry 2004, 75, 6-12. [PubMed]

31. Sboros, V. Response of contrast agents to ultrasound. Adv. Drug Deliv. Rev. 2008, 60, 1117-1136. [CrossRef] [PubMed]

32. Weissleder, R. Molecular imaging in cancer. Science 2006, 312, 1168-1171. [CrossRef] [PubMed]

33. Hogemann, D.; Basilion, J.P.; Weissleder, R. Molecular imaging techniques in magnetic resonance imaging and nuclear imaging. Der Radiologe 2001, 41, 116-120. [PubMed]

34. Liao, L.D.; Lin, C.T.; Shih, Y.Y.I.; Duong, T.Q.; Lai, H.Y.; Wang, P.H.; Wu, R.; Tsang, S.; Chang, J.-Y.; Li, M.-L.; et al. Transcranial imaging of functional cerebral hemodynamic changes in single blood vessels using in vivo photoacoustic microscopy. J. Cereb. Blood Flow Metab. 2012, 32, 938-951. [CrossRef]

35. Liu, Y.H.; Xu, Y.; Liao, L.D.; Chan, K.C.; Thakor, N.V. A Handheld Real-Time Photoacoustic Imaging System for Animal Neurological Disease Models: From Simulation to Realization. Sensors 2018, 18, 4081. [CrossRef]

36. Mason, R.P. Oxygen breathing challenge—the simplest theranostic. Theranostics 2017, 7, 3873-3875. [CrossRef]

37. Yao, D.K.; Zhang, C.; Maslov, K.; Wang, L.V. Photoacoustic measurement of the Gruneisen parameter of tissue. J. Biomed. Opt. 2014, 19, 17007. [CrossRef]

38. Scholz, A.; Detjen, K.; Schulz, P.; Rexin, A.; Steller, L.; Amthauer, H.; Wiedenmann, B. Development of orthotopic mouse models of pancreatic cancer, hepatocellular carcinoma and neuroendocrine tumors for preclinical evaluation of new substances. Ann. Oncol. $2007,18,37$.

39. Schneider, C.A.; Rasband, W.S.; Eliceiri, K.W. NIH Image to ImageJ: 25 years of image analysis. Nat. Methods 2012, 9, 671-675. [CrossRef]

40. Li, M.L.; Oh, J.T.; Xie, X.Y.; Ku, G.; Wang, W.; Li, C.; Lungu, G.; Stoica, G.; Wang, L.V. Simultaneous molecular and hypoxia imaging of brain tumors in vivo using spectroscopic photoacoustic tomography. Proc. IEEE 2008, 96, 481-489.

41. Laufer, J.; Elwell, C.; Delpy, D.; Beard, P. In vitro measurements of absolute blood oxygen saturation using pulsed near-infrared photoacoustic spectroscopy: Accuracy and resolution. Phys. Med. Biol. 2005, 50, 4409-4428. [CrossRef]

42. Cox, B.; Laufer, J.G.; Arridge, S.R.; Beard, P.C. Quantitative spectroscopic photoacoustic imaging: A review. J. Biomed. Opt. 2012, 17, 061202. [CrossRef]

43. Kim, S.; Chen, Y.S.; Luke, G.P.; Emelianov, S.Y. In vivo three-dimensional spectroscopic photoacoustic imaging for monitoring nanoparticle delivery. Biomed. Opt. Express 2011, 2, 2540-2550. [CrossRef] 
44. Yin, L.; Wang, Q.; Zhang, Q.Z.; Jiang, H.B. Tomographic imaging of absolute optical absorption coefficient in turbid media using combined photoacoustic and diffusing light measurements. Opt. Lett. 2007, 32, 2556-2558. [CrossRef]

45. Chen, Z.; Yang, S.; Xing, D. In vivo detection of hemoglobin oxygen saturation and carboxyhemoglobin saturation with multiwavelength photoacoustic microscopy. Opt. Lett 2012, 37, 3414-3416. [CrossRef]

46. Lakshman, M.; Needles, A. Screening and quantification of the tumor microenvironment with micro-ultrasound and photoacoustic imaging. Nat. Methods 2015, 12, iii-v. [CrossRef]

47. Vaccaro, V.; Sperduti, I.; Milella, M. FOLFIRINOX versus gemcitabine for metastatic pancreatic cancer. N. Engl. J. Med. 2011, 365, 768-769.

48. Phung, C.D.; Tran, T.H.; Pham, L.M.; Nguyen, H.T.; Jeong, J.H.; Yong, C.S.; Kim, J.O. Current developments in nanotechnology for improved cancer treatment, focusing on tumor hypoxia. J. Control. Release 2020, 324, 413-429. [CrossRef]

49. von Hoff, D.D.; Ervin, T.; Arena, F.P.; Chiorean, E.G.; Infante, J.; Moore, M.; Seay, T.; Tjulandin, S.A.; Ma, W.W.; Saleh, M.N.; et al. Increased survival in pancreatic cancer with nab-paclitaxel plus gemcitabine. N. Engl J. Med. 2013, 369, 1691-1703. [CrossRef]

50. Cai, X.L.; Bandla, A.; Chuan, C.K.; Magarajah, G.; Liao, L.D.; Teh, D.B.L.; Kennedy, B.K.; Thakor, N.V.; Liu, B. Identifying glioblastoma margins using dual-targeted organic nanoparticles for efficient in vivo fluorescence image-guided photothermal therapy. Mater. Horiz. 2019, 6, 311-317. [CrossRef]

51. Liu, J.; Cai, X.L.; Pan, H.C.; Bandla, A.; Chuan, C.K.; Wang, S.W.; Thakor, N.; Lio, L.-D.; Liu, B. Molecular Engineering of Photoacoustic Performance by Chalcogenide Variation in Conjugated Polymer Nanoparticles for Brain Vascular Imaging. Small 2018, 14, 1703732. [CrossRef]

52. Gerling, M.; Zhao, Y.; Nania, S.; Norberg, K.J.; Verbeke, C.S.; Englert, B.; Kuiper, R.V.; Bergström, Å.; Hassan, M.; Neesse, A.; et al. Real-time assessment of tissue hypoxia in vivo with combined photoacoustics and high-frequency ultrasound. Theranostics 2014, 4, 604-613. [CrossRef] 